\title{
Domestic Violence and Football in Glasgow: Are Reference Points Relevant? Online Appendix
}

\author{
Alex Dickson ${ }^{\dagger}$, Colin Jennings ${ }^{\S}$ and Gary Koop ${ }^{1 \dagger}$ \\ ${ }^{\dagger}$ Department of Economics, University of Strathclyde \\ $\S$ Department of Political Economy, King's College London
}

May 11, 2015

${ }^{1}$ Corresponding author. Economics, University of Strathclyde, Glasgow, G4 0GE; email: gary.koop@strath.ac.uk; tel: +44(0)141548 3862. 


\section{A Alternative Specifications}

In this section we present the results of alternative specifications to check the robustness of our results presented in Tables 4 and 5. Towards the end of this section we present the results of our models estimated using negative binomial regression and OLS methods. With the latter, the log of the number of domestic violence incidents is the dependent variable. These give almost identical results to those estimated using Poisson regression methods presented in the paper. We also consider the effect of changing the probability of winning threshold that governs our classification of expectations. In the following section we present results using a mixed effects model.

\section{A.1 Altering the winning probability threshold}

In this subsection we consider the effect on our results of changing the probability that defines whether a match is predicted to be close or whether the team is expected to win. The aim of setting this threshold is to separate the matches a team plays to clearly identify those where it is expected to win, allowing us to clearly classify unexpected outcomes. In the paper this threshold is 0.7 . We believe this reflects the reality of non-Old Firm matches in the SPL (the average winning probability for Celtic is 0.77 and for Rangers is 0.75 ), and allows us to deduce that if a team is expected to win and then either draws or loses fans are likely to perceive this as disappointing relative to their expectations. That said, the selection of 0.7 is rather arbitrary so in this section we consider thresholds of 0.6 and 0.8 .

When the threshold reduces to 0.6 , the results from which are presented in Table A.1 (note that only the results in Table 5 change as the threshold does not affect the explanatory variables in Table 4), fewer matches are classified as tight. The estimates of model (B1') change very little except for the coefficient on rtightdraw increasing in magnitude due to the reduced number of observations falling into that category. Our original results suggest that in important matches where Celtic or Rangers are favourite to win a non-Old Firm match and the outcome is a draw there is an increase in domestic violence that is significantly different from a draw when a match is predicted to be tight. When the threshold is reduced to 0.6 the coefficients on favdraw become insignificant for both teams, and there are no observations where the match is predicted to be tight and the outcome is a draw. As such, we lose the basis of the 'upset non-win' effect we identified previously. The reason for this is that with the lower threshold all matches where the teams draw, even those where the winning probability is relatively low and so a draw might not be that unexpected, are grouped into a single category so the coefficient estimate is the average effect associated with 


\begin{tabular}{|c|c|c|c|c|c|}
\hline & $\begin{array}{c}\left(\mathrm{B} 1^{\prime}\right) \\
\text { All matches }\end{array}$ & $\begin{array}{c}\left(\mathrm{B} 2^{\prime}\right) \\
\text { Importance }\end{array}$ & $\begin{array}{c}\text { (B3') } \\
\text { Extended importance }\end{array}$ & $\begin{array}{c}\left(\mathrm{B} 4{ }^{\prime}\right) \\
\text { Traditional rival }\end{array}$ & $\begin{array}{c}\left(\mathrm{B} 5{ }^{\prime}\right) \\
\text { Referee active }\end{array}$ \\
\hline cfavwin & $\begin{array}{l}-0.0252 \\
(0.0378)\end{array}$ & $\begin{array}{l}-0.0266 \\
(0.0359)\end{array}$ & $\begin{array}{l}-0.0426 \\
(0.0385)\end{array}$ & $\begin{array}{l}-0.0153 \\
(0.0367)\end{array}$ & $\begin{array}{l}-0.0411 \\
(0.0399)\end{array}$ \\
\hline ctightwin & $\begin{array}{r}-0.0289 \\
(0.0611)\end{array}$ & $\begin{array}{l}-0.0371 \\
(0.0602)\end{array}$ & $\begin{array}{l}-0.0821 \\
(0.0733)\end{array}$ & $\begin{array}{l}0.00234 \\
(0.0626)\end{array}$ & $\begin{array}{l}\text { omitted } \\
(.)\end{array}$ \\
\hline cfavdraw & $\begin{array}{l}-0.0508 \\
(0.0395)\end{array}$ & $\begin{array}{c}-0.0681^{*} \\
(0.0405)\end{array}$ & $\begin{array}{c}-0.0812^{*} \\
(0.0440)\end{array}$ & $\begin{array}{l}-0.0762^{*} \\
(0.0414)\end{array}$ & $\begin{array}{l}-0.0774 \\
(0.0526)\end{array}$ \\
\hline ctightdraw & $\begin{array}{l}-0.0312 \\
(0.0480)\end{array}$ & $\begin{array}{l}-0.0420 \\
(0.0456)\end{array}$ & $\begin{array}{l}\text { omitted } \\
(.)\end{array}$ & $\begin{array}{l}\text { omitted } \\
(.)\end{array}$ & $\begin{array}{l}\text { omitted } \\
(.)\end{array}$ \\
\hline cfavlose & $\begin{array}{l}-0.0289 \\
(0.0556)\end{array}$ & $\begin{array}{l}-0.0614 \\
(0.0542)\end{array}$ & $\begin{array}{l}-0.0791 \\
(0.0660)\end{array}$ & $\begin{array}{l}-0.00179 \\
(0.0595)\end{array}$ & $\begin{array}{l}0.00914 \\
(0.0834)\end{array}$ \\
\hline ctightlose & $\begin{array}{l}-0.0546 \\
(0.0449)\end{array}$ & $\begin{array}{l}-0.0609 \\
(0.0432)\end{array}$ & $\begin{array}{r}-0.0758^{*} \\
(0.0451)\end{array}$ & $\begin{array}{l}-0.0470 \\
(0.0439)\end{array}$ & $\begin{array}{l}-0.101^{*} \\
(0.0588)\end{array}$ \\
\hline rfavwin & $\begin{array}{l}-0.0305 \\
(0.0291)\end{array}$ & $\begin{array}{l}-0.0355 \\
(0.0288)\end{array}$ & $\begin{array}{l}-0.0417 \\
(0.0298)\end{array}$ & $\begin{array}{l}-0.0365 \\
(0.0286)\end{array}$ & $\begin{array}{l}-0.0115 \\
(0.0314)\end{array}$ \\
\hline rtightwin & $\begin{array}{l}0.00131 \\
(0.0480)\end{array}$ & $\begin{array}{c}-0.00194 \\
(0.0478)\end{array}$ & $\begin{array}{c}-0.00708 \\
(0.0512)\end{array}$ & $\begin{array}{c}0.0288 \\
(0.0498)\end{array}$ & $\begin{array}{l}-0.0638 \\
(0.0613)\end{array}$ \\
\hline rfavdraw & $\begin{array}{l}-0.0166 \\
(0.0387)\end{array}$ & $\begin{array}{l}-0.0167 \\
(0.0396)\end{array}$ & $\begin{array}{l}-0.0155 \\
(0.0421)\end{array}$ & $\begin{array}{c}-0.0141 \\
(0.0418)\end{array}$ & $\begin{array}{r}-0.0140 \\
(0.0494)\end{array}$ \\
\hline rtightdraw & $\begin{array}{c}0.141^{*} \\
(0.0770)\end{array}$ & $\begin{array}{c}0.141^{*} \\
(0.0769)\end{array}$ & $\begin{array}{l}0.196^{* *} \\
(0.0782)\end{array}$ & $\begin{array}{c}0.0519 \\
(0.0803)\end{array}$ & $\begin{array}{l}0.239^{* * *} \\
(0.0428)\end{array}$ \\
\hline rfavlose & $\begin{array}{l}-0.0458 \\
(0.0418)\end{array}$ & $\begin{array}{l}-0.0532 \\
(0.0429)\end{array}$ & $\begin{array}{l}-0.0529 \\
(0.0464)\end{array}$ & $\begin{array}{l}-0.0781 \\
(0.0516)\end{array}$ & $\begin{array}{l}-0.136^{* *} \\
(0.0625)\end{array}$ \\
\hline rtightlose & $\begin{array}{c}0.0371 \\
(0.0460)\end{array}$ & $\begin{array}{c}0.0316 \\
(0.0463)\end{array}$ & $\begin{array}{c}0.0274 \\
(0.0463)\end{array}$ & $\begin{array}{c}0.0551 \\
(0.0495)\end{array}$ & $\begin{array}{l}-0.0322 \\
(0.0389)\end{array}$ \\
\hline cfavwin_sal & & $\begin{array}{l}-0.00911 \\
(0.0702)\end{array}$ & $\begin{array}{r}-0.00943 \\
(0.0477)\end{array}$ & $\begin{array}{l}-0.0454 \\
(0.0387)\end{array}$ & $\begin{array}{l}-0.0210 \\
(0.0397)\end{array}$ \\
\hline ctightwin_sal & & $\begin{array}{c}\text { omitted } \\
(.)\end{array}$ & $\begin{array}{c}0.0117 \\
(0.0664)\end{array}$ & $\begin{array}{l}-0.0507 \\
(0.0800)\end{array}$ & $\begin{array}{l}-0.0313 \\
(0.0620)\end{array}$ \\
\hline cfavdraw_sal & & $\begin{array}{c}0.206 \\
(0.132)\end{array}$ & $\begin{array}{c}0.0195 \\
(0.0781)\end{array}$ & $\begin{array}{c}0.0543 \\
(0.0521)\end{array}$ & $\begin{array}{l}-0.0483 \\
(0.0429)\end{array}$ \\
\hline ctightdraw_sal & & $\begin{array}{l}\text { omitted } \\
(.)\end{array}$ & $\begin{array}{c}-0.0902^{*} \\
(0.0537)\end{array}$ & $\begin{array}{c}-0.0309 \\
(0.0390)\end{array}$ & $\begin{array}{l}-0.0258 \\
(0.0510)\end{array}$ \\
\hline cfavlose_sal & & $\begin{array}{c}0.198 \\
(0.126)\end{array}$ & $\begin{array}{c}0.0358 \\
(0.0837)\end{array}$ & $\begin{array}{c}-0.0764 \\
(0.0797)\end{array}$ & $\begin{array}{c}-0.0696 \\
(0.0576)\end{array}$ \\
\hline ctightlose_sal & & $\begin{array}{l}\text { omitted } \\
(.)\end{array}$ & $\begin{array}{l}\text { omitted } \\
(.)\end{array}$ & $\begin{array}{c}-0.0413 \\
(0.0527)\end{array}$ & $\begin{array}{l}-0.0557 \\
(0.0492)\end{array}$ \\
\hline rfavwin_sal & & $\begin{array}{c}0.0384 \\
(0.0664)\end{array}$ & $\begin{array}{c}-0.00494 \\
(0.0389)\end{array}$ & $\begin{array}{c}-0.0465 \\
(0.0393)\end{array}$ & $\begin{array}{c}-0.0367 \\
(0.0310)\end{array}$ \\
\hline rtightwin_sal & & $\begin{array}{c}\text { omitted } \\
(.)\end{array}$ & $\begin{array}{l}-0.0116 \\
(0.0355)\end{array}$ & $\begin{array}{c}-0.113^{*} \\
(0.0636)\end{array}$ & $\begin{array}{c}0.0633 \\
(0.0580)\end{array}$ \\
\hline rfardraw_sal & & $\begin{array}{c}0.00170 \\
(0.0867)\end{array}$ & $\begin{array}{c}-0.0621 \\
(0.0559)\end{array}$ & $\begin{array}{c}-0.0485 \\
(0.0426)\end{array}$ & $\begin{array}{c}-0.00611 \\
(0.0430)\end{array}$ \\
\hline rtightdraw_sal & & $\begin{array}{c}\text { omitted } \\
(.)\end{array}$ & $\begin{array}{c}-0.0229 \\
(0.120)\end{array}$ & $\begin{array}{c}0.153 \\
(0.110)\end{array}$ & $\begin{array}{c}0.120 \\
(0.0994)\end{array}$ \\
\hline rfavlose_sal & & $\begin{array}{l}0.181^{* * *} \\
(0.0639)\end{array}$ & $\begin{array}{c}-0.0125 \\
(0.0929)\end{array}$ & $\begin{array}{c}-0.00420 \\
(0.0451)\end{array}$ & $\begin{array}{c}0.0166 \\
(0.0464)\end{array}$ \\
\hline rtightlose_sal & & $\begin{array}{c}\text { omitted } \\
(.)\end{array}$ & $\begin{array}{c}\text { omitted } \\
(.)\end{array}$ & $\begin{array}{c}-0.0202 \\
(0.0555)\end{array}$ & $\begin{array}{c}0.0805 \\
(0.0532)\end{array}$ \\
\hline oldfirm_draw & $\begin{array}{c}0.387^{* * *} \\
(0.112)\end{array}$ & $\begin{array}{c}0.387^{* * *} \\
(0.112)\end{array}$ & $\begin{array}{c}0.388^{* * *} \\
(0.112)\end{array}$ & $\begin{array}{c}0.386^{* * *} \\
(0.112)\end{array}$ & $\begin{array}{c}0.386^{* * *} \\
(0.112)\end{array}$ \\
\hline oldfirm_close & $\begin{array}{l}0.410^{* * *} \\
(0.0536)\end{array}$ & $\begin{array}{c}0.409^{* * *} \\
(0.0536)\end{array}$ & $\begin{array}{l}0.410^{* * *} \\
(0.0537)\end{array}$ & $\begin{array}{l}0.409^{* * *} \\
(0.0535)\end{array}$ & $\begin{array}{l}0.410^{* * *} \\
(0.0536)\end{array}$ \\
\hline oldfirm_rlose & $\begin{array}{l}0.285^{* * *} \\
(0.0464)\end{array}$ & $\begin{array}{c}0.284^{* * *} \\
(0.0464)\end{array}$ & $\begin{array}{c}0.285^{* * *} \\
(0.0465)\end{array}$ & $\begin{array}{c}0.284^{* * *} \\
(0.0464)\end{array}$ & $\begin{array}{l}0.284^{* * *} \\
(0.0465)\end{array}$ \\
\hline cvstr & $\begin{array}{c}-0.00925 \\
(0.0217)\end{array}$ & $\begin{array}{c}-0.00333 \\
(0.0209)\end{array}$ & $\begin{array}{c}-0.00378 \\
(0.0213)\end{array}$ & & $\begin{array}{c}-0.00842 \\
(0.0225)\end{array}$ \\
\hline rustr & $\begin{array}{c}-0.0139 \\
(0.0204)\end{array}$ & $\begin{array}{c}-0.0172 \\
(0.0197)\end{array}$ & $\begin{array}{c}-0.0177 \\
(0.0209)\end{array}$ & & $\begin{array}{c}-0.0197 \\
(0.0204)\end{array}$ \\
\hline chome & $\begin{array}{c}0.0193 \\
(0.0344)\end{array}$ & $\begin{array}{c}0.0238 \\
(0.0331)\end{array}$ & $\begin{array}{c}0.0298 \\
(0.0356)\end{array}$ & $\begin{array}{c}0.0155 \\
(0.0335)\end{array}$ & $\begin{array}{c}0.0242 \\
(0.0346)\end{array}$ \\
\hline rhome & $\begin{array}{c}0.0262 \\
(0.0303)\end{array}$ & $\begin{array}{c}0.0316 \\
(0.0301)\end{array}$ & $\begin{array}{c}0.0347 \\
(0.0304)\end{array}$ & $\begin{array}{c}0.0289 \\
(0.0300)\end{array}$ & $\begin{array}{c}0.0209 \\
(0.0300)\end{array}$ \\
\hline ctv & $\begin{array}{l}0.0603^{*} \\
(0.0346)\end{array}$ & $\begin{array}{l}0.0652^{*} \\
(0.0335)\end{array}$ & $\begin{array}{l}0.0795^{* *} \\
(0.0354)\end{array}$ & $\begin{array}{c}0.0532 \\
(0.0340)\end{array}$ & $\begin{array}{l}0.0647^{*} \\
(0.0357)\end{array}$ \\
\hline rtv & $\begin{array}{c}0.0453 \\
(0.0294)\end{array}$ & $\begin{array}{l}0.0485^{*} \\
(0.0294)\end{array}$ & $\begin{array}{c}0.0611^{* *} \\
(0.0292)\end{array}$ & $\begin{array}{l}0.0532^{*} \\
(0.0293)\end{array}$ & $\begin{array}{c}0.0373 \\
(0.0294)\end{array}$ \\
\hline cimp & $\begin{array}{l}0.0837^{*} \\
(0.0446)\end{array}$ & & & $\begin{array}{l}0.0800^{*} \\
(0.0435)\end{array}$ & $\begin{array}{l}0.0807^{*} \\
(0.0454)\end{array}$ \\
\hline rimp & $\begin{array}{c}0.0825 \\
(0.0507)\end{array}$ & & & $\begin{array}{c}0.0711 \\
(0.0488)\end{array}$ & $\begin{array}{c}0.0817 \\
(0.0508)\end{array}$ \\
\hline oldfirm_imp & $\begin{array}{c}0.106 \\
(0.0685) \\
\end{array}$ & $\begin{array}{c}0.106 \\
(0.0685) \\
\end{array}$ & $\begin{array}{c}0.106 \\
(0.0686) \\
\end{array}$ & $\begin{array}{c}0.106 \\
(0.0686) \\
\end{array}$ & $\begin{array}{c}0.106 \\
(0.0686)\end{array}$ \\
\hline intercept & $\begin{array}{l}4.230^{* * *} \\
(0.0143)\end{array}$ & $\begin{array}{l}4.231^{* * *} \\
(0.0143)\end{array}$ & $\begin{array}{l}4.230^{* * *} \\
(0.0144)\end{array}$ & $\begin{array}{l}4.229^{* * *} \\
(0.0143)\end{array}$ & $\begin{array}{l}4.230^{* * *} \\
(0.0143)\end{array}$ \\
\hline$N$ & 3200 & 3200 & 3200 & 3200 & 3200 \\
\hline
\end{tabular}

Table A.1: The effects of match outcomes relative to expectations when the winning probability threshold is reduced to 0.6 . 
a draw. This makes sense, as fans in these lower probability matches are less disappointed with a draw than if the winning probability is higher. The only other change is that the coefficient on rfavlose becomes positive and significant which results from matches where Rangers lost that were classed as being tight are now classed as Rangers being favourite to win.

As such, lowering the threshold probability means unexpected results cannot be so easily identified by our categorical variables (e.g. favdraw) which means we no longer find an upset non-win effect in important matches.

When the threshold is increased to 0.8 , the results of which are in Table A.2, a team is deemed to be favourite only when the pre-match betting probability is particularly high, so for such matches the team losing or drawing is on average more unexpected for fans than when the threshold is 0.7. Considering all matches in model (B1"), this change results in a significantly positive coefficient for Celtic when they are predicted to win and then lose; just as when we focussed on important matches we uncovered an upset non-win effect, when we focus on matches where the pre-match winning probability is particularly high - so losing such a match is particularly unexpected - we find such an effect. It seems as though there are some observations for Celtic where the winning probability is particularly high, the team loses and there is a high level of domestic violence. Model (B2") suggests that the majority of this effect is attributable to important matches ( $\mathrm{p}$-value for a test of equality between the coefficients on cfavlose in important matches and non-important matches is 0.097).

The conclusion that unexpectedly bad results in important games are associated with higher levels of domestic violence under this specification remains true for Celtic but the higher threshold probability means that for Rangers there are no observations where the team was favourite and the result was a draw so no conclusion can be made.

Our original model reported negative coefficients for many match category variables, suggesting a reduction in domestic violence associated with football. Some coefficient estimates with this specification suggest that this is significant, even when the team loses. This is a rather strange finding but further suggests that reference dependent behaviour coupled with loss aversion is not an appropriate description of fans' behaviour in general.

To summarise, the findings of our study suggest that unexpectedly bad results are not associated with increased levels of domestic violence in general. If we focus on particularly salient matches at the end of the season where the title is still being contested we do find an effect, but this is based on very few observations. If we relax the definition of 'expected to win' this result is lost. If we strengthen it so that a team is deemed favourite only when the winning probability is particularly high and so losing such a match would be particularly 
unexpected there is evidence of an upset loss effect for Celtic fans. The overall conclusion remains that unexpected results are associated with increased levels of domestic violence only in a small subset of matches that occur at the end of the season where the title is still being contested. 


\begin{tabular}{|c|c|c|c|c|c|}
\hline & $\begin{array}{c}\text { (B1") } \\
\text { All matches }\end{array}$ & $\begin{array}{c}\text { (B2") } \\
\text { Importance }\end{array}$ & $\begin{array}{c}(\mathrm{B} 3 ") \\
\text { Extended importance }\end{array}$ & $\begin{array}{c}\text { (B4") } \\
\text { Traditional rival }\end{array}$ & $\begin{array}{c}(\mathrm{B} 5 ") \\
\text { Referee active }\end{array}$ \\
\hline cfavwin & $\begin{array}{c}-0.0194 \\
(0.0400)\end{array}$ & $\begin{array}{l}-0.0107 \\
(0.0385)\end{array}$ & $\begin{array}{l}-0.0220 \\
(0.0388)\end{array}$ & $\begin{array}{l}-0.0127 \\
(0.0390)\end{array}$ & $\begin{array}{l}-0.0367 \\
(0.0408)\end{array}$ \\
\hline ctightwin & $\begin{array}{l}-0.0247 \\
(0.0369)\end{array}$ & $\begin{array}{r}-0.00620 \\
(0.0363)\end{array}$ & $\begin{array}{l}-0.0224 \\
(0.0354)\end{array}$ & $\begin{array}{r}-0.00632 \\
(0.0359)\end{array}$ & $\begin{array}{l}-0.0224 \\
(0.0458)\end{array}$ \\
\hline cfavdraw & $\begin{array}{c}-0.0330 \\
(0.0470)\end{array}$ & $\begin{array}{l}-0.0541 \\
(0.0470)\end{array}$ & $\begin{array}{c}-0.0659 \\
(0.0483)\end{array}$ & $\begin{array}{r}-0.0704 \\
(0.0483)\end{array}$ & $\begin{array}{l}0.00989 \\
(0.0635)\end{array}$ \\
\hline ctightdraw & $\begin{array}{l}-0.0498 \\
(0.0449)\end{array}$ & $\begin{array}{l}-0.0381 \\
(0.0457)\end{array}$ & $\begin{array}{l}-0.0442 \\
(0.0480)\end{array}$ & $\begin{array}{c}-0.0731 \\
(0.0464)\end{array}$ & $\begin{array}{c}-0.109 \\
(0.0665)\end{array}$ \\
\hline cfavlose & $\begin{array}{l}0.128^{* *} \\
(0.0606)\end{array}$ & $\begin{array}{c}0.102^{*} \\
(0.0537)\end{array}$ & $\begin{array}{l}0.0988^{* *} \\
(0.0454)\end{array}$ & $\begin{array}{l}0.148^{* *} \\
(0.0631)\end{array}$ & $\begin{array}{l}0.204^{* *} \\
(0.0825)\end{array}$ \\
\hline ctightlose & $\begin{array}{l}-0.122^{* *} \\
(0.0513)\end{array}$ & $\begin{array}{l}-0.110^{* *} \\
(0.0531)\end{array}$ & $\begin{array}{l}-0.101^{*} \\
(0.0585)\end{array}$ & $\begin{array}{l}-0.112^{* *} \\
(0.0568)\end{array}$ & $\begin{array}{l}-0.153^{*} \\
(0.0817)\end{array}$ \\
\hline rfavwin & $\begin{array}{r}-0.00361 \\
(0.0314)\end{array}$ & $\begin{array}{r}-0.00595 \\
(0.0320)\end{array}$ & $\begin{array}{c}-0.00672 \\
(0.0336)\end{array}$ & $\begin{array}{r}-0.00357 \\
(0.0325)\end{array}$ & $\begin{array}{l}0.00803 \\
(0.0348)\end{array}$ \\
\hline rtightwin & $\begin{array}{l}-0.0375 \\
(0.0302)\end{array}$ & $\begin{array}{l}-0.0465 \\
(0.0289)\end{array}$ & $\begin{array}{c}-0.0532^{*} \\
(0.0307)\end{array}$ & $\begin{array}{l}-0.0448 \\
(0.0301)\end{array}$ & $\begin{array}{l}-0.0356 \\
(0.0330)\end{array}$ \\
\hline rfavdraw & $\begin{array}{r}-0.00238 \\
(0.0610)\end{array}$ & $\begin{array}{r}-0.00743 \\
(0.0606)\end{array}$ & $\begin{array}{c}-0.0000512 \\
(0.0699)\end{array}$ & $\begin{array}{l}0.000828 \\
(0.0598)\end{array}$ & $\begin{array}{r}-0.0739 \\
(0.0862)\end{array}$ \\
\hline rtightdraw & $\begin{array}{l}0.00412 \\
(0.0386)\end{array}$ & $\begin{array}{l}0.00648 \\
(0.0392)\end{array}$ & $\begin{array}{c}0.0130 \\
(0.0414)\end{array}$ & $\begin{array}{r}-0.00223 \\
(0.0442)\end{array}$ & $\begin{array}{c}0.0189 \\
(0.0502)\end{array}$ \\
\hline rfavlose & $\begin{array}{l}0.00598 \\
(0.0584)\end{array}$ & $\begin{array}{l}0.00230 \\
(0.0581)\end{array}$ & $\begin{array}{c}0.0304 \\
(0.0556)\end{array}$ & $\begin{array}{l}0.00477 \\
(0.0669)\end{array}$ & $\begin{array}{l}-0.0612 \\
(0.0627)\end{array}$ \\
\hline rtightlose & $\begin{array}{l}-0.0252 \\
(0.0420)\end{array}$ & $\begin{array}{l}-0.0333 \\
(0.0424)\end{array}$ & $\begin{array}{l}-0.0361 \\
(0.0452)\end{array}$ & $\begin{array}{l}-0.0515 \\
(0.0525)\end{array}$ & $\begin{array}{c}-0.149^{* *} \\
(0.0720)\end{array}$ \\
\hline cfavwin_sal & & $\begin{array}{l}0.0700 \\
(0.109)\end{array}$ & $\begin{array}{c}0.0148 \\
(0.0509)\end{array}$ & $\begin{array}{l}-0.0265 \\
(0.0537)\end{array}$ & $\begin{array}{c}-0.00631 \\
(0.0435)\end{array}$ \\
\hline ctightwin_sal & & $\begin{array}{l}-0.0282 \\
(0.0605)\end{array}$ & $\begin{array}{l}0.00700 \\
(0.0455)\end{array}$ & $\begin{array}{c}-0.0401 \\
(0.0378)\end{array}$ & $\begin{array}{l}-0.0268 \\
(0.0381)\end{array}$ \\
\hline cfavdraw_sal & & $\begin{array}{l}0.393^{* * *} \\
(0.0864)\end{array}$ & $\begin{array}{c}0.208 \\
(0.156)\end{array}$ & $\begin{array}{c}0.150 \\
(0.0996)\end{array}$ & $\begin{array}{l}-0.0788 \\
(0.0563)\end{array}$ \\
\hline ctightdraw_sal & & $\begin{array}{c}0.0205 \\
(0.0333)\end{array}$ & $\begin{array}{c}-0.0584 \\
(0.0491)\end{array}$ & $\begin{array}{c}0.0335 \\
(0.0539)\end{array}$ & $\begin{array}{c}-0.0172 \\
(0.0451)\end{array}$ \\
\hline cfavlose_sal & & $\begin{array}{l}0.316^{* *} \\
(0.128)\end{array}$ & $\begin{array}{l}0.187^{* *} \\
(0.0922)\end{array}$ & $\begin{array}{l}0.0965 \\
(0.107)\end{array}$ & $\begin{array}{c}0.0447 \\
(0.0483)\end{array}$ \\
\hline ctightlose_sal & & $\begin{array}{c}-0.0421 \\
(0.0340)\end{array}$ & $\begin{array}{c}-0.172^{* * *} \\
(0.0614)\end{array}$ & $\begin{array}{c}-0.122^{*} \\
(0.0734)\end{array}$ & $\begin{array}{c}-0.119^{* *} \\
(0.0579)\end{array}$ \\
\hline rfavwin_sal & & $\begin{array}{c}-0.00326 \\
(0.130)\end{array}$ & $\begin{array}{c}-0.0118 \\
(0.0501)\end{array}$ & $\begin{array}{c}-0.0509 \\
(0.0405)\end{array}$ & $\begin{array}{c}-0.0185 \\
(0.0361)\end{array}$ \\
\hline rtightwin_sal & & $\begin{array}{c}0.0277 \\
(0.0685)\end{array}$ & $\begin{array}{c}0.00301 \\
(0.0419)\end{array}$ & $\begin{array}{c}-0.0402 \\
(0.0403)\end{array}$ & $\begin{array}{c}-0.0366 \\
(0.0336)\end{array}$ \\
\hline rfavdraw_sal & & $\begin{array}{c}\text { omitted } \\
(.)\end{array}$ & $\begin{array}{c}-0.0592 \\
(0.0443)\end{array}$ & $\begin{array}{c}\text { omitted } \\
(.)\end{array}$ & $\begin{array}{c}0.0279 \\
(0.0669)\end{array}$ \\
\hline rtightdraw_sal & & $\begin{array}{c}-0.00869 \\
(0.0871)\end{array}$ & $\begin{array}{c}-0.0349 \\
(0.0610)\end{array}$ & $\begin{array}{c}-0.00151 \\
(0.0475)\end{array}$ & $\begin{array}{c}0.000660 \\
(0.0430)\end{array}$ \\
\hline rfavlose_sal & & $\begin{array}{c}\text { omitted } \\
(.)\end{array}$ & $\begin{array}{c}-0.216^{* * *} \\
(0.0317)\end{array}$ & $\begin{array}{c}0.000435 \\
(0.0955)\end{array}$ & $\begin{array}{l}0.133^{* * *} \\
(0.0402)\end{array}$ \\
\hline rtightlose_sal & & $\begin{array}{l}0.193^{* * *} \\
(0.0591)\end{array}$ & $\begin{array}{c}0.0567 \\
(0.0720)\end{array}$ & $\begin{array}{l}0.00960 \\
(0.0402)\end{array}$ & $\begin{array}{c}0.0264 \\
(0.0453)\end{array}$ \\
\hline oldfirm_draw & $\begin{array}{c}0.387^{* * *} \\
(0.112)\end{array}$ & $\begin{array}{c}0.387^{* * *} \\
(0.112)\end{array}$ & $\begin{array}{c}0.388^{* * *} \\
(0.112)\end{array}$ & $\begin{array}{c}0.386^{* * *} \\
(0.112)\end{array}$ & $\begin{array}{c}0.385^{* * *} \\
(0.112)\end{array}$ \\
\hline oldfirm_close & $\begin{array}{l}0.410^{* * *} \\
(0.0536)\end{array}$ & $\begin{array}{c}0.409^{* * *} \\
(0.0536)\end{array}$ & $\begin{array}{l}0.410^{* * *} \\
(0.0537)\end{array}$ & $\begin{array}{c}0.409^{* * *} \\
(0.0536)\end{array}$ & $\begin{array}{l}0.410^{* * *} \\
(0.0536)\end{array}$ \\
\hline oldfirm_rlose & $\begin{array}{l}0.286^{* * *} \\
(0.0464)\end{array}$ & $\begin{array}{c}0.285^{* * *} \\
(0.0464)\end{array}$ & $\begin{array}{c}0.286^{* * *} \\
(0.0464)\end{array}$ & $\begin{array}{c}0.286^{* * *} \\
(0.0463)\end{array}$ & $\begin{array}{l}0.284^{* * *} \\
(0.0466)\end{array}$ \\
\hline cvstr & $\begin{array}{c}0.00169 \\
(0.0220)\end{array}$ & $\begin{array}{c}-0.00251 \\
(0.0214)\end{array}$ & $\begin{array}{c}-0.00814 \\
(0.0216)\end{array}$ & & $\begin{array}{c}-0.00132 \\
(0.0226)\end{array}$ \\
\hline rvstr & $\begin{array}{c}-0.00186 \\
(0.0208)\end{array}$ & $\begin{array}{c}-0.00460 \\
(0.0204)\end{array}$ & $\begin{array}{c}-0.00719 \\
(0.0209)\end{array}$ & & $\begin{array}{l}-0.0105 \\
(0.0204)\end{array}$ \\
\hline chome & $\begin{array}{l}0.00758 \\
(0.0346)\end{array}$ & $\begin{array}{c}0.00142 \\
(0.0347)\end{array}$ & $\begin{array}{l}0.00699 \\
(0.0340)\end{array}$ & $\begin{array}{l}0.00568 \\
(0.0339)\end{array}$ & $\begin{array}{c}0.0107 \\
(0.0346)\end{array}$ \\
\hline rhome & $\begin{array}{l}0.00494 \\
(0.0296)\end{array}$ & $\begin{array}{c}0.0100 \\
(0.0295)\end{array}$ & $\begin{array}{c}0.0119 \\
(0.0302)\end{array}$ & $\begin{array}{l}0.00805 \\
(0.0299)\end{array}$ & $\begin{array}{l}0.00897 \\
(0.0296)\end{array}$ \\
\hline ctv & $\begin{array}{c}0.0652^{* *} \\
(0.0330)\end{array}$ & $\begin{array}{c}0.0528 \\
(0.0327)\end{array}$ & $\begin{array}{l}0.0648^{* *} \\
(0.0312)\end{array}$ & $\begin{array}{c}0.0552^{*} \\
(0.0319)\end{array}$ & $\begin{array}{c}0.0660^{*} \\
(0.0339)\end{array}$ \\
\hline rtv & $\begin{array}{l}0.0512^{*} \\
(0.0293)\end{array}$ & $\begin{array}{l}0.0556^{*} \\
(0.0288)\end{array}$ & $\begin{array}{c}0.0635^{* *} \\
(0.0291)\end{array}$ & $\begin{array}{c}0.0563^{*} \\
(0.0296)\end{array}$ & $\begin{array}{c}0.0469 \\
(0.0294)\end{array}$ \\
\hline cimp & $\begin{array}{l}0.0668^{*} \\
(0.0406)\end{array}$ & & & $\begin{array}{c}0.0629 \\
(0.0399)\end{array}$ & $\begin{array}{c}0.0630 \\
(0.0401)\end{array}$ \\
\hline rimp & $\begin{array}{c}0.0759 \\
(0.0489)\end{array}$ & & & $\begin{array}{c}0.0638 \\
(0.0471)\end{array}$ & $\begin{array}{c}0.0705 \\
(0.0487)\end{array}$ \\
\hline oldfirm_imp & $\begin{array}{c}0.106 \\
(0.0689) \\
\end{array}$ & $\begin{array}{c}0.106 \\
(0.0689) \\
\end{array}$ & $\begin{array}{c}0.105 \\
(0.0688) \\
\end{array}$ & $\begin{array}{c}0.106 \\
(0.0688) \\
\end{array}$ & $\begin{array}{c}0.107 \\
(0.0691) \\
\end{array}$ \\
\hline intercept & $\begin{array}{l}4.229^{* * *} \\
(0.0143)\end{array}$ & $\begin{array}{l}4.230^{* * *} \\
(0.0143)\end{array}$ & $\begin{array}{l}4.230^{* * *} \\
(0.0143)\end{array}$ & $\begin{array}{l}4.229^{* * *} \\
(0.0143)\end{array}$ & $\begin{array}{l}4.228^{* * *} \\
(0.0144)\end{array}$ \\
\hline$N$ & 3200 & 3200 & 3200 & 3200 & 3200 \\
\hline
\end{tabular}

Table A.2: The effects of match outcomes relative to expectations when the winning probability threshold is increased to 0.8 . 


\section{A.2 Negative Binomial Regressions}

\begin{tabular}{|c|c|c|c|c|c|c|}
\hline & (A1nb) & (A2nb) & (A3nb) & (A4nb) & (A5nb) & $\overline{(\mathrm{A} 6 \mathrm{nb})}$ \\
\hline conly & -0.00488 & -0.00204 & $-0.0702^{*}$ & -0.0526 & $-0.0625^{*}$ & \\
\hline & $(0.0124)$ & & & $(0.0352)$ & & \\
\hline ronly & -0.00457 & -0.00378 & -0.0460 & -0.0393 & -0.0351 & \\
\hline candr & $\begin{array}{l}(0.0132) \\
0.142^{\text {*** }} \\
(0.0211)\end{array}$ & $(0.0146)$ & $(0.0299)$ & $(0.0286)$ & $(0.0296)$ & \\
\hline candr $\neg$ & & $\begin{array}{c}0.0596^{* * *} \\
(0.0204)\end{array}$ & $\begin{array}{l}-0.0527 \\
(0.0452)\end{array}$ & $\begin{array}{l}-0.0208 \\
(0.0448)\end{array}$ & $\begin{array}{c}-0.0230 \\
(0.0484)\end{array}$ & \\
\hline oldfirm & & $\begin{array}{l}0.366^{* * *} \\
(0.0344)\end{array}$ & $\begin{array}{l}0.369^{* * *} \\
(0.0351)\end{array}$ & $\begin{array}{l}0.354^{* * *} \\
(0.0392)\end{array}$ & $\begin{array}{l}0.401^{* * *} \\
(0.0701)\end{array}$ & \\
\hline custr & & $\begin{array}{c}-0.00360 \\
(0.0225)\end{array}$ & $\begin{array}{c}-0.00208 \\
(0.0217)\end{array}$ & $\begin{array}{c}-0.00694 \\
(0.0210)\end{array}$ & $\begin{array}{r}-0.00948 \\
(0.0214)\end{array}$ & $\begin{array}{r}-0.00771 \\
(0.0216)\end{array}$ \\
\hline rvstr & & $\begin{array}{c}0.000644 \\
(0.0224)\end{array}$ & $\begin{array}{c}-0.00129 \\
(0.0216)\end{array}$ & $\begin{array}{c}-0.00402 \\
(0.0200)\end{array}$ & $\begin{array}{r}-0.00320 \\
(0.0203)\end{array}$ & $\begin{array}{r}-0.00474 \\
(0.0206)\end{array}$ \\
\hline chome & & & $\begin{array}{c}0.0437 \\
(0.0340)\end{array}$ & $\begin{array}{c}0.0244 \\
(0.0321)\end{array}$ & $\begin{array}{c}0.0266 \\
(0.0323)\end{array}$ & $\begin{array}{c}0.0205 \\
(0.0324)\end{array}$ \\
\hline rhome & & & $\begin{array}{c}0.0253 \\
(0.0297)\end{array}$ & $\begin{array}{c}0.0160 \\
(0.0285)\end{array}$ & $\begin{array}{c}0.0155 \\
(0.0285)\end{array}$ & $\begin{array}{c}0.0176 \\
(0.0282)\end{array}$ \\
\hline ctv & & & $\begin{array}{l}0.0847^{* *} \\
(0.0343)\end{array}$ & $\begin{array}{c}0.0561^{*} \\
(0.0329)\end{array}$ & $\begin{array}{c}0.0557^{*} \\
(0.0333)\end{array}$ & $\begin{array}{c}0.0572^{*} \\
(0.0330)\end{array}$ \\
\hline rtv & & & $\begin{array}{l}0.0599^{* *} \\
(0.0288)\end{array}$ & $\begin{array}{c}0.0384 \\
(0.0279)\end{array}$ & $\begin{array}{c}0.0387 \\
(0.0281)\end{array}$ & $\begin{array}{c}0.0463^{*} \\
(0.0274)\end{array}$ \\
\hline cimp & & & & $\begin{array}{l}0.0895^{* *} \\
(0.0421)\end{array}$ & $\begin{array}{l}0.0884^{* *} \\
(0.0427)\end{array}$ & $\begin{array}{c}0.0841^{*} \\
(0.0452)\end{array}$ \\
\hline rimp & & & & $\begin{array}{l}0.0981^{*} \\
(0.0508)\end{array}$ & $\begin{array}{c}0.0978^{*} \\
(0.0510)\end{array}$ & $\begin{array}{c}0.0929^{*} \\
(0.0530)\end{array}$ \\
\hline oldfirm_imp & & & & $\begin{array}{c}0.0900 \\
(0.0698)\end{array}$ & $\begin{array}{c}0.117 \\
(0.0731)\end{array}$ & $\begin{array}{c}0.107 \\
(0.0731)\end{array}$ \\
\hline cref & & & & & $\begin{array}{c}0.0153 \\
(0.0191)\end{array}$ & \\
\hline rref & & & & & $\begin{array}{c}-0.0100 \\
(0.0207)\end{array}$ & \\
\hline oldfirm_ref & & & & & $\begin{array}{c}-0.0736 \\
(0.0824)\end{array}$ & \\
\hline oldfirm_draw & & & & & & $\begin{array}{c}0.394^{* * *} \\
(0.112)\end{array}$ \\
\hline oldfirm_close & & & & & & $\begin{array}{l}0.425^{* * *} \\
(0.0531)\end{array}$ \\
\hline oldfirm_rlose & & & & & & $\begin{array}{c}0.291^{* * *} \\
(0.0480)\end{array}$ \\
\hline cwin & & & & & & $\begin{array}{l}-0.0241 \\
(0.0357)\end{array}$ \\
\hline cdraw & & & & & & $\begin{array}{l}-0.0415 \\
(0.0379)\end{array}$ \\
\hline close & & & & & & $\begin{array}{c}-0.0301 \\
(0.0504)\end{array}$ \\
\hline rwin & & & & & & $\begin{array}{c}-0.0257 \\
(0.0271)\end{array}$ \\
\hline rdraw & & & & & & $\begin{array}{c}0.00246 \\
(0.0350)\end{array}$ \\
\hline rlose & & & & & & $\begin{array}{c}-0.0270 \\
(0.0383)\end{array}$ \\
\hline intercept & $\begin{array}{l}4.222^{* * *} \\
(0.0140)\end{array}$ & $\begin{array}{l}4.228^{* * *} \\
(0.0138)\end{array}$ & $\begin{array}{l}4.234^{* * *} \\
(0.0139)\end{array}$ & $\begin{array}{l}4.232^{* * *} \\
(0.0139)\end{array}$ & $\begin{array}{l}4.233^{* * *} \\
(0.0139)\end{array}$ & $\begin{array}{l}4.229^{* * *} \\
(0.0139)\end{array}$ \\
\hline$N$ & 3200 & 3200 & 3200 & 3200 & 3200 & 3200 \\
\hline
\end{tabular}

${ }^{*} p<0.10,{ }^{* *} p<0.05,{ }^{* * *} p<0.01$

Table A.3: Negative binomial regression estimates of specifications A1-A6. 


\begin{tabular}{|c|c|c|c|c|c|}
\hline & $\begin{array}{c}\text { (B1nb) } \\
\text { All matches }\end{array}$ & $\begin{array}{l}\text { (B2nb) } \\
\text { Importance }\end{array}$ & $\begin{array}{c}\text { (B3nb) } \\
\text { Extended importance }\end{array}$ & $\begin{array}{c}\text { (B4nb) } \\
\text { Traditional rival }\end{array}$ & $\begin{array}{c}\text { (B5nb) } \\
\text { Referee active }\end{array}$ \\
\hline cfavwin & $\begin{array}{c}-0.0292 \\
(0.0370)\end{array}$ & $\begin{array}{c}-0.0208 \\
(0.0363)\end{array}$ & $\begin{array}{c}-0.0442 \\
(0.0365)\end{array}$ & $\begin{array}{l}-0.0173 \\
(0.0369)\end{array}$ & $\begin{array}{c}-0.0416 \\
(0.0389)\end{array}$ \\
\hline ctightwin & $\begin{array}{c}-0.0201 \\
(0.0405)\end{array}$ & $\begin{array}{c}-0.00659 \\
(0.0406)\end{array}$ & $\begin{array}{c}-0.0410 \\
(0.0396)\end{array}$ & $\begin{array}{c}-0.0249 \\
(0.0434)\end{array}$ & $\begin{array}{c}-0.0459 \\
(0.0706)\end{array}$ \\
\hline cfavdraw & $\begin{array}{l}-0.0517 \\
(0.0441)\end{array}$ & $\begin{array}{l}-0.0613 \\
(0.0447)\end{array}$ & $\begin{array}{l}-0.0796^{*} \\
(0.0453)\end{array}$ & $\begin{array}{c}-0.0810^{*} \\
(0.0453)\end{array}$ & $\begin{array}{c}-0.0691 \\
(0.0592)\end{array}$ \\
\hline ctightdraw & $\begin{array}{c}-0.0325 \\
(0.0440)\end{array}$ & $\begin{array}{l}-0.0241 \\
(0.0462)\end{array}$ & $\begin{array}{l}-0.0400 \\
(0.0537)\end{array}$ & $\begin{array}{c}-0.0610 \\
(0.0511)\end{array}$ & $\begin{array}{l}-0.0623 \\
(0.0924)\end{array}$ \\
\hline cfavlose & $\begin{array}{l}-0.0134 \\
(0.0607)\end{array}$ & $\begin{array}{l}-0.0429 \\
(0.0613)\end{array}$ & $\begin{array}{l}-0.0655 \\
(0.0795)\end{array}$ & $\begin{array}{l}-0.0270 \\
(0.0699)\end{array}$ & $\begin{array}{l}0.00391 \\
(0.0982)\end{array}$ \\
\hline ctightlose & $\begin{array}{l}-0.0598 \\
(0.0629)\end{array}$ & $\begin{array}{l}-0.0515 \\
(0.0623)\end{array}$ & $\begin{array}{l}-0.0753 \\
(0.0616)\end{array}$ & $\begin{array}{l}0.00348 \\
(0.0547)\end{array}$ & $\begin{array}{c}0.000369 \\
(0.0715)\end{array}$ \\
\hline rfavwin & $\begin{array}{l}-0.0308 \\
(0.0310)\end{array}$ & $\begin{array}{l}-0.0364 \\
(0.0307)\end{array}$ & $\begin{array}{l}-0.0369 \\
(0.0324)\end{array}$ & $\begin{array}{l}-0.0351 \\
(0.0311)\end{array}$ & $\begin{array}{c}-0.0140 \\
(0.0358)\end{array}$ \\
\hline rtightwin & $\begin{array}{l}-0.0206 \\
(0.0302)\end{array}$ & $\begin{array}{l}-0.0328 \\
(0.0295)\end{array}$ & $\begin{array}{l}-0.0499 \\
(0.0333)\end{array}$ & $\begin{array}{l}-0.0181 \\
(0.0310)\end{array}$ & $\begin{array}{l}-0.0213 \\
(0.0342)\end{array}$ \\
\hline rfavdraw & $\begin{array}{r}-0.00240 \\
(0.0438)\end{array}$ & $\begin{array}{l}-0.0140 \\
(0.0446)\end{array}$ & $\begin{array}{l}-0.0156 \\
(0.0503)\end{array}$ & $\begin{array}{l}0.00371 \\
(0.0469)\end{array}$ & $\begin{array}{r}-0.00916 \\
(0.0586)\end{array}$ \\
\hline rtightdraw & $\begin{array}{l}0.00643 \\
(0.0432)\end{array}$ & $\begin{array}{c}0.0189 \\
(0.0440)\end{array}$ & $\begin{array}{c}0.0387 \\
(0.0448)\end{array}$ & $\begin{array}{l}-0.0169 \\
(0.0502)\end{array}$ & $\begin{array}{c}0.0173 \\
(0.0641)\end{array}$ \\
\hline rfavlose & $\begin{array}{c}-0.0198 \\
(0.0471)\end{array}$ & $\begin{array}{l}-0.0275 \\
(0.0468)\end{array}$ & $\begin{array}{l}-0.0175 \\
(0.0523)\end{array}$ & $\begin{array}{c}-0.0382 \\
(0.0517)\end{array}$ & $\begin{array}{c}-0.0965^{* *} \\
(0.0461)\end{array}$ \\
\hline rtightlose & $\begin{array}{c}-0.0330 \\
(0.0502)\end{array}$ & $\begin{array}{c}-0.0444 \\
(0.0519)\end{array}$ & $\begin{array}{c}-0.0476 \\
(0.0530)\end{array}$ & $\begin{array}{l}-0.0643 \\
(0.0828)\end{array}$ & $\begin{array}{l}-0.226 \\
(0.156)\end{array}$ \\
\hline cfavwin_sal & & $\begin{array}{c}0.0172 \\
(0.0825)\end{array}$ & $\begin{array}{l}-0.0140 \\
(0.0480)\end{array}$ & $\begin{array}{l}-0.0769^{*} \\
(0.0408)\end{array}$ & $\begin{array}{l}-0.0255 \\
(0.0397)\end{array}$ \\
\hline ctightwin_sal & & $\begin{array}{c}-0.0190 \\
(0.0982)\end{array}$ & $\begin{array}{l}0.00309 \\
(0.0638)\end{array}$ & $\begin{array}{r}-0.00779 \\
(0.0457)\end{array}$ & $\begin{array}{l}-0.0250 \\
(0.0427)\end{array}$ \\
\hline cfavdraw_sal & & $\begin{array}{l}0.340^{* *} \\
(0.150)\end{array}$ & $\begin{array}{l}0.0482 \\
(0.147)\end{array}$ & $\begin{array}{c}0.132^{*} \\
(0.0703)\end{array}$ & $\begin{array}{l}-0.0455 \\
(0.0502)\end{array}$ \\
\hline ctightdraw_sal & & $\begin{array}{c}0.0228 \\
(0.0342)\end{array}$ & $\begin{array}{l}-0.0644 \\
(0.0444)\end{array}$ & $\begin{array}{c}-0.00480 \\
(0.0475)\end{array}$ & $\begin{array}{l}-0.0343 \\
(0.0436)\end{array}$ \\
\hline cfavlose_sal & & $\begin{array}{c}0.213 \\
(0.132)\end{array}$ & $\begin{array}{c}0.0413 \\
(0.0878)\end{array}$ & $\begin{array}{c}0.0368 \\
(0.0946)\end{array}$ & $\begin{array}{c}-0.0456 \\
(0.0467)\end{array}$ \\
\hline ctightlose_sal & & $\begin{array}{l}\text { omitted } \\
(.)\end{array}$ & $\begin{array}{c}\text { omitted } \\
(.)\end{array}$ & $\begin{array}{c}-0.124 \\
(0.0860)\end{array}$ & $\begin{array}{c}-0.0984 \\
(0.0755)\end{array}$ \\
\hline rfavwin_sal & & $\begin{array}{c}0.0319 \\
(0.0762)\end{array}$ & $\begin{array}{l}-0.0127 \\
(0.0475)\end{array}$ & $\begin{array}{c}-0.0335 \\
(0.0441)\end{array}$ & $\begin{array}{c}-0.0370 \\
(0.0323)\end{array}$ \\
\hline rtightwin_sal & & $\begin{array}{c}0.0762 \\
(0.136)\end{array}$ & $\begin{array}{c}0.0387 \\
(0.0407)\end{array}$ & $\begin{array}{c}-0.0661 \\
(0.0451)\end{array}$ & $\begin{array}{c}-0.00510 \\
(0.0377)\end{array}$ \\
\hline rfavdraw_sal & & $\begin{array}{l}0.243^{* * *} \\
(0.0293)\end{array}$ & $\begin{array}{c}0.0322 \\
(0.0589)\end{array}$ & $\begin{array}{c}-0.0350 \\
(0.0599)\end{array}$ & $\begin{array}{l}0.00940 \\
(0.0497)\end{array}$ \\
\hline rtightdraw_sal & & $\begin{array}{c}-0.0826^{* * *} \\
(0.0391)\end{array}$ & $\begin{array}{c}-0.105 \\
(0.0702)\end{array}$ & $\begin{array}{l}0.00925 \\
(0.0597)\end{array}$ & $\begin{array}{c}0.00992 \\
(0.0513)\end{array}$ \\
\hline rfavlose_sal & & $\begin{array}{c}\text { omitted } \\
(.)\end{array}$ & $\begin{array}{c}-0.132^{*} \\
(0.0678)\end{array}$ & $\begin{array}{c}0.0510 \\
(0.0864)\end{array}$ & $\begin{array}{c}0.0521 \\
(0.0635)\end{array}$ \\
\hline rtightlose_sal & & $\begin{array}{c}0.151^{* *} \\
(0.0747)\end{array}$ & $\begin{array}{l}0.153^{* * * *} \\
(0.0521)\end{array}$ & $\begin{array}{c}-0.0265 \\
(0.0383)\end{array}$ & $\begin{array}{l}0.00926 \\
(0.0435)\end{array}$ \\
\hline oldfirm_draw & $\begin{array}{c}0.394^{* * *} \\
(0.112)\end{array}$ & $\begin{array}{c}0.394^{* * *} \\
(0.112)\end{array}$ & $\begin{array}{c}0.394^{* * * *} \\
(0.112)\end{array}$ & $\begin{array}{c}0.393^{* * *} \\
(0.112)\end{array}$ & $\begin{array}{c}0.394^{* * *} \\
(0.112)\end{array}$ \\
\hline oldfirm_close & $\begin{array}{l}0.425^{* * *} \\
(0.0530)\end{array}$ & $\begin{array}{l}0.425^{* * *} \\
(0.0531)\end{array}$ & $\begin{array}{l}0.425^{* * *} \\
(0.0530)\end{array}$ & $\begin{array}{c}0.424^{* * *} \\
(0.0530)\end{array}$ & $\begin{array}{l}0.425^{* * *} \\
(0.0531)\end{array}$ \\
\hline oldfirm_rlose & $\begin{array}{l}0.291^{* * *} \\
(0.0480)\end{array}$ & $\begin{array}{c}0.290^{* * *} \\
(0.0481)\end{array}$ & $\begin{array}{c}0.291^{* * *} \\
(0.0480)\end{array}$ & $\begin{array}{c}0.290^{* * *} \\
(0.0479)\end{array}$ & $\begin{array}{c}0.290^{* * *} \\
(0.0481)\end{array}$ \\
\hline cvstr & $\begin{array}{c}-0.00825 \\
(0.0221)\end{array}$ & $\begin{array}{l}-0.0107 \\
(0.0217)\end{array}$ & $\begin{array}{c}-0.00931 \\
(0.0217)\end{array}$ & & $\begin{array}{c}0.000774 \\
(0.0235)\end{array}$ \\
\hline rvstr & $\begin{array}{c}-0.00451 \\
(0.0214)\end{array}$ & $\begin{array}{c}-0.00601 \\
(0.0201)\end{array}$ & $\begin{array}{c}-0.00868 \\
(0.0218)\end{array}$ & & $\begin{array}{c}-0.0132 \\
(0.0211)\end{array}$ \\
\hline chome & $\begin{array}{c}0.0249 \\
(0.0336)\end{array}$ & $\begin{array}{c}0.0211 \\
(0.0340)\end{array}$ & $\begin{array}{c}0.0364 \\
(0.0340)\end{array}$ & $\begin{array}{c}0.0219 \\
(0.0340)\end{array}$ & $\begin{array}{c}0.0279 \\
(0.0344)\end{array}$ \\
\hline rhome & $\begin{array}{c}0.0214 \\
(0.0321)\end{array}$ & $\begin{array}{c}0.0284 \\
(0.0315)\end{array}$ & $\begin{array}{c}0.0300 \\
(0.0331)\end{array}$ & $\begin{array}{c}0.0221 \\
(0.0320)\end{array}$ & $\begin{array}{c}0.0178 \\
(0.0323)\end{array}$ \\
\hline ctv & $\begin{array}{c}0.0606^{*} \\
(0.0336)\end{array}$ & $\begin{array}{l}0.0549^{*} \\
(0.0333)\end{array}$ & $\begin{array}{l}0.0757^{* *} \\
(0.0326)\end{array}$ & $\begin{array}{l}0.0564^{*} \\
(0.0335)\end{array}$ & $\begin{array}{c}0.0644^{*} \\
(0.0351)\end{array}$ \\
\hline rtv & $\begin{array}{l}0.0459^{*} \\
(0.0271)\end{array}$ & $\begin{array}{l}0.0507^{*} \\
(0.0269)\end{array}$ & $\begin{array}{c}0.0620^{* *} \\
(0.0277)\end{array}$ & $\begin{array}{c}0.0518^{*} \\
(0.0276)\end{array}$ & $\begin{array}{c}0.0400 \\
(0.0273)\end{array}$ \\
\hline cimp & $\begin{array}{l}0.0799^{*} \\
(0.0451)\end{array}$ & & & $\begin{array}{l}0.0774^{*} \\
(0.0440)\end{array}$ & $\begin{array}{l}0.0780^{*} \\
(0.0470)\end{array}$ \\
\hline rimp & $\begin{array}{l}0.0941^{*} \\
(0.0530)\end{array}$ & & & $\begin{array}{c}0.0816 \\
(0.0519)\end{array}$ & $\begin{array}{c}0.0939^{*} \\
(0.0536)\end{array}$ \\
\hline oldfirm_imp & $\begin{array}{c}0.107 \\
(0.0731)\end{array}$ & $\begin{array}{c}0.107 \\
(0.0733)\end{array}$ & $\begin{array}{c}0.106 \\
(0.0731)\end{array}$ & $\begin{array}{c}0.107 \\
(0.0731)\end{array}$ & $\begin{array}{c}0.107 \\
(0.0733) \\
\end{array}$ \\
\hline intercept & $\begin{array}{l}4.230^{* * *} \\
(0.0140)\end{array}$ & $\begin{array}{l}4.231^{* * *} \\
(0.0140)\end{array}$ & $\begin{array}{l}4.230^{* * *} \\
(0.0140)\end{array}$ & $\begin{array}{l}4.230^{* * *} \\
(0.0140)\end{array}$ & $\begin{array}{l}4.230^{* * *} \\
(0.0140)\end{array}$ \\
\hline$N$ & 3200 & 3200 & 3200 & 3200 & 3200 \\
\hline
\end{tabular}

Table A.4: Negative binomial regression estimates of specifications B1-B5. 


\section{A.3 OLS Regressions}

\begin{tabular}{|c|c|c|c|c|c|c|}
\hline & (A1ols) & (A2ols) & (A3ols) & (A4ols) & (A5ols) & (A6ols) \\
\hline \multirow[t]{2}{*}{ conly } & 0.00348 & 0.00666 & $-0.0620^{*}$ & -0.0479 & $-0.0587^{*}$ & \\
\hline & $(0.0127)$ & $(0.0133)$ & $(0.0359)$ & $(0.0334)$ & $(0.0347)$ & \\
\hline \multirow[t]{2}{*}{ ronly } & 0.00147 & 0.00130 & -0.0385 & -0.0331 & -0.0295 & \\
\hline & $(0.0134)$ & $(0.0149)$ & $(0.0292)$ & $(0.0284)$ & $(0.0294)$ & \\
\hline \multirow[t]{2}{*}{ candr } & $0.139^{* * *}$ & & & & & \\
\hline & $(0.0201)$ & & & & & \\
\hline \multirow{2}{*}{ candr $\neg$} & & $0.0639^{* * *}$ & -0.0450 & -0.0177 & -0.0219 & \\
\hline & & $(0.0202)$ & $(0.0442)$ & $(0.0442)$ & $(0.0478)$ & \\
\hline \multirow[t]{2}{*}{ oldfirm } & & $0.372^{* * *}$ & $0.375^{* * *}$ & $0.360^{* * *}$ & $0.407^{* * *}$ & \\
\hline & & $(0.0325)$ & $(0.0331)$ & $(0.0365)$ & $(0.0643)$ & \\
\hline \multirow[t]{2}{*}{ cvstr } & & -0.00558 & -0.00319 & -0.00755 & -0.0106 & -0.00779 \\
\hline & & $(0.0227)$ & $(0.0221)$ & $(0.0218)$ & $(0.0223)$ & $(0.0223)$ \\
\hline \multirow[t]{2}{*}{ rvstr } & & 0.00592 & 0.00438 & 0.00208 & 0.00259 & 0.00287 \\
\hline & & $(0.0215)$ & $(0.0206)$ & $(0.0194)$ & $(0.0195)$ & $(0.0199)$ \\
\hline \multirow[t]{2}{*}{ chome } & & & 0.0441 & 0.0276 & 0.0298 & 0.0246 \\
\hline & & & $(0.0320)$ & $(0.0306)$ & $(0.0309)$ & $(0.0309)$ \\
\hline \multirow[t]{2}{*}{ rhome } & & & 0.0216 & 0.0132 & 0.0125 & 0.0158 \\
\hline & & & $(0.0290)$ & $(0.0285)$ & $(0.0285)$ & $(0.0282)$ \\
\hline \multirow[t]{2}{*}{$\operatorname{ctv}$} & & & $0.0829^{* *}$ & $0.0589^{*}$ & $0.0581^{*}$ & $0.0583^{*}$ \\
\hline & & & $(0.0324)$ & $(0.0316)$ & $(0.0322)$ & $(0.0316)$ \\
\hline \multirow[t]{2}{*}{$\mathrm{rtv}$} & & & $0.0578^{* *}$ & 0.0391 & 0.0393 & $0.0453^{*}$ \\
\hline & & & $(0.0283)$ & $(0.0277)$ & $(0.0280)$ & $(0.0271)$ \\
\hline \multirow[t]{2}{*}{ cimp } & & & & $0.0838^{* *}$ & $0.0822^{*}$ & $0.0785^{*}$ \\
\hline & & & & $(0.0416)$ & $(0.0422)$ & $(0.0442)$ \\
\hline \multirow[t]{2}{*}{ rimp } & & & & $0.0927^{*}$ & $0.0929^{*}$ & 0.0872 \\
\hline & & & & $(0.0541)$ & $(0.0543)$ & $(0.0557)$ \\
\hline \multirow[t]{2}{*}{ oldfirm_imp } & & & & 0.0905 & 0.117 & 0.111 \\
\hline & & & & $(0.0708)$ & $(0.0739)$ & $(0.0708)$ \\
\hline \multirow[t]{2}{*}{ cref } & & & & & 0.0173 & \\
\hline & & & & & $(0.0192)$ & \\
\hline \multirow[t]{2}{*}{ rref } & & & & & -0.00795 & \\
\hline & & & & & $(0.0205)$ & \\
\hline \multirow[t]{2}{*}{ oldfirm_ref } & & & & & -0.0727 & \\
\hline & & & & & $(0.0760)$ & \\
\hline oldfirm_draw & & & & & & $0.396^{* * *}$ \\
\hline oldfirm_close & & & & & & $0.431^{* * *}$ \\
\hline & & & & & & $(0.0508)$ \\
\hline oldfirm_rlose & & & & & & $0.301^{* * *}$ \\
\hline & & & & & & $(0.0442)$ \\
\hline cwin & & & & & & -0.0218 \\
\hline & & & & & & $(0.0339)$ \\
\hline cdraw & & & & & & -0.0351 \\
\hline & & & & & & $(0.0362)$ \\
\hline close & & & & & & -0.0370 \\
\hline & & & & & & $(0.0506)$ \\
\hline rwin & & & & & & -0.0219 \\
\hline & & & & & & $(0.0270)$ \\
\hline rdraw & & & & & & 0.00521 \\
\hline & & & & & & $(0.0347)$ \\
\hline rlose & & & & & & -0.0261 \\
\hline & & & & & & $(0.0394)$ \\
\hline intercept & $4.204^{* * *}$ & $4.208^{* * *}$ & $4.215^{* * *}$ & $4.214^{* * *}$ & $4.214^{* * *}$ & $4.211^{* * *}$ \\
\hline & $(0.0138)$ & $(0.0137)$ & $(0.0138)$ & $(0.0138)$ & $(0.0139)$ & $(0.0138)$ \\
\hline$N$ & 3200 & 3200 & 3200 & 3200 & 3200 & 3200 \\
\hline
\end{tabular}

Table A.5: OLS regression estimates of specifications A1-A6; dependent variable is the log of the number of domestic violence incidents. 


\begin{tabular}{|c|c|c|c|c|c|}
\hline & $\begin{array}{c}\text { (B1ols) } \\
\text { All matches }\end{array}$ & $\begin{array}{c}\text { (B2ols) } \\
\text { Importance }\end{array}$ & $\begin{array}{c}\text { (B3ols) } \\
\text { Extended importance }\end{array}$ & $\begin{array}{c}\text { (B4ols) } \\
\text { Traditional rival }\end{array}$ & $\begin{array}{c}\text { (B5ols) } \\
\text { Referee active }\end{array}$ \\
\hline cfavwin & $\begin{array}{l}-0.0275 \\
(0.0352)\end{array}$ & $\begin{array}{c}-0.0212 \\
(0.0348)\end{array}$ & $\begin{array}{l}-0.0440 \\
(0.0353)\end{array}$ & $\begin{array}{l}-0.0179 \\
(0.0360)\end{array}$ & $\begin{array}{l}-0.0413 \\
(0.0373)\end{array}$ \\
\hline ctightwin & $\begin{array}{c}-0.0138 \\
(0.0394)\end{array}$ & $\begin{array}{c}-0.00286 \\
(0.0398)\end{array}$ & $\begin{array}{c}-0.0345 \\
(0.0391)\end{array}$ & $\begin{array}{c}-0.0187 \\
(0.0431)\end{array}$ & $\begin{array}{c}-0.0448 \\
(0.0750)\end{array}$ \\
\hline cfavdraw & $\begin{array}{l}-0.0463 \\
(0.0424)\end{array}$ & $\begin{array}{l}-0.0556 \\
(0.0429)\end{array}$ & $\begin{array}{l}-0.0695 \\
(0.0439)\end{array}$ & $\begin{array}{l}-0.0758^{*} \\
(0.0439)\end{array}$ & $\begin{array}{l}-0.0653 \\
(0.0567)\end{array}$ \\
\hline ctightdraw & $\begin{array}{l}-0.0206 \\
(0.0440)\end{array}$ & $\begin{array}{c}-0.0160 \\
(0.0467)\end{array}$ & $\begin{array}{l}-0.0309 \\
(0.0550)\end{array}$ & $\begin{array}{l}-0.0534 \\
(0.0528)\end{array}$ & $\begin{array}{l}-0.0591 \\
(0.0921)\end{array}$ \\
\hline cfavlose & $\begin{array}{l}-0.0249 \\
(0.0615)\end{array}$ & $\begin{array}{l}-0.0533 \\
(0.0642)\end{array}$ & $\begin{array}{l}-0.0776 \\
(0.0856)\end{array}$ & $\begin{array}{l}-0.0515 \\
(0.0722)\end{array}$ & $\begin{array}{l}-0.0241 \\
(0.102)\end{array}$ \\
\hline ctightlose & $\begin{array}{l}-0.0578 \\
(0.0659)\end{array}$ & $\begin{array}{l}-0.0523 \\
(0.0657)\end{array}$ & $\begin{array}{l}-0.0736 \\
(0.0654)\end{array}$ & $\begin{array}{l}0.00805 \\
(0.0548)\end{array}$ & $\begin{array}{l}0.00253 \\
(0.0727)\end{array}$ \\
\hline rfavwin & $\begin{array}{l}-0.0260 \\
(0.0310)\end{array}$ & $\begin{array}{l}-0.0305 \\
(0.0309)\end{array}$ & $\begin{array}{l}-0.0299 \\
(0.0321)\end{array}$ & $\begin{array}{l}-0.0318 \\
(0.0313)\end{array}$ & $\begin{array}{l}-0.0109 \\
(0.0365)\end{array}$ \\
\hline rtightwin & $\begin{array}{l}-0.0158 \\
(0.0296)\end{array}$ & $\begin{array}{l}-0.0276 \\
(0.0293)\end{array}$ & $\begin{array}{l}-0.0451 \\
(0.0329)\end{array}$ & $\begin{array}{l}-0.0164 \\
(0.0307)\end{array}$ & $\begin{array}{l}-0.0110 \\
(0.0329)\end{array}$ \\
\hline rfavdraw & $\begin{array}{c}0.0103 \\
(0.0429)\end{array}$ & $\begin{array}{r}-0.00226 \\
(0.0438)\end{array}$ & $\begin{array}{r}-0.00281 \\
(0.0495)\end{array}$ & $\begin{array}{c}0.0168 \\
(0.0455)\end{array}$ & $\begin{array}{l}0.00680 \\
(0.0575)\end{array}$ \\
\hline rtightdraw & $\begin{array}{c}-0.00295 \\
(0.0423)\end{array}$ & $\begin{array}{l}0.00767 \\
(0.0438)\end{array}$ & $\begin{array}{c}0.0310 \\
(0.0436)\end{array}$ & $\begin{array}{l}-0.0229 \\
(0.0500)\end{array}$ & $\begin{array}{l}0.00631 \\
(0.0653)\end{array}$ \\
\hline rfavlose & $\begin{array}{r}-0.0150 \\
(0.0480)\end{array}$ & $\begin{array}{l}-0.0221 \\
(0.0479)\end{array}$ & $\begin{array}{r}-0.00922 \\
(0.0541)\end{array}$ & $\begin{array}{l}-0.0364 \\
(0.0525)\end{array}$ & $\begin{array}{c}-0.0964^{* *} \\
(0.0469)\end{array}$ \\
\hline rtightlose & $\begin{array}{r}-0.0390 \\
(0.0532)\end{array}$ & $\begin{array}{l}-0.0512 \\
(0.0553)\end{array}$ & $\begin{array}{l}-0.0525 \\
(0.0565)\end{array}$ & $\begin{array}{l}-0.0859 \\
(0.0899)\end{array}$ & $\begin{array}{l}-0.251^{*} \\
(0.152)\end{array}$ \\
\hline cfavwin_sal & & $\begin{array}{l}0.0166 \\
(0.0776)\end{array}$ & $\begin{array}{l}-0.00821 \\
(0.0466)\end{array}$ & $\begin{array}{c}-0.0800^{* *} \\
(0.0404)\end{array}$ & $\begin{array}{l}-0.0249 \\
(0.0384)\end{array}$ \\
\hline ctightwin_sal & & $\begin{array}{l}-0.0234 \\
(0.0928)\end{array}$ & $\begin{array}{l}0.00450 \\
(0.0650)\end{array}$ & $\begin{array}{l}-0.0103 \\
(0.0466)\end{array}$ & $\begin{array}{l}-0.0189 \\
(0.0415)\end{array}$ \\
\hline cfavdraw_sal & & $\begin{array}{l}0.330^{* *} \\
(0.150)\end{array}$ & $\begin{array}{l}0.00364 \\
(0.145)\end{array}$ & $\begin{array}{c}0.138^{*} \\
(0.0719)\end{array}$ & $\begin{array}{l}-0.0419 \\
(0.0496)\end{array}$ \\
\hline ctightdraw_sal & & $\begin{array}{c}0.0407 \\
(0.0331)\end{array}$ & $\begin{array}{l}-0.0475 \\
(0.0465)\end{array}$ & $\begin{array}{l}0.00761 \\
(0.0478)\end{array}$ & $\begin{array}{l}-0.0197 \\
(0.0424)\end{array}$ \\
\hline cfavlose_sal & & $\begin{array}{c}0.204 \\
(0.133)\end{array}$ & $\begin{array}{c}0.0274 \\
(0.0877)\end{array}$ & $\begin{array}{c}0.0482 \\
(0.0913)\end{array}$ & $\begin{array}{l}-0.0392 \\
(0.0446)\end{array}$ \\
\hline ctightlose_sal & & $\begin{array}{l}0 \\
(.)\end{array}$ & $\begin{array}{c}\text { omitted } \\
(.)\end{array}$ & $\begin{array}{l}-0.129 \\
(0.0921)\end{array}$ & $\begin{array}{l}-0.0981 \\
(0.0801)\end{array}$ \\
\hline rfavwin_sal & & $\begin{array}{c}0.0262 \\
(0.0824)\end{array}$ & $\begin{array}{l}-0.0131 \\
(0.0486)\end{array}$ & $\begin{array}{l}-0.0268 \\
(0.0420)\end{array}$ & $\begin{array}{l}-0.0314 \\
(0.0326)\end{array}$ \\
\hline rtightwin_sal & & $\begin{array}{c}0.0982 \\
(0.138)\end{array}$ & $\begin{array}{c}0.0505 \\
(0.0407)\end{array}$ & $\begin{array}{l}-0.0480 \\
(0.0438)\end{array}$ & $\begin{array}{l}-0.00557 \\
(0.0384)\end{array}$ \\
\hline rfavdraw_sal & & $\begin{array}{l}0.270^{* * *} \\
(0.0290)\end{array}$ & $\begin{array}{c}0.0503 \\
(0.0621)\end{array}$ & $\begin{array}{l}-0.0266 \\
(0.0600)\end{array}$ & $\begin{array}{c}0.0183 \\
(0.0487)\end{array}$ \\
\hline rtightdraw_sal & & $\begin{array}{c}-0.0682^{*} \\
(0.0395)\end{array}$ & $\begin{array}{l}-0.100 \\
(0.0731)\end{array}$ & $\begin{array}{l}0.00368 \\
(0.0522)\end{array}$ & $\begin{array}{l}0.00151 \\
(0.0487)\end{array}$ \\
\hline rfavlose_sal & & $\begin{array}{l}0 \\
(.)\end{array}$ & $\begin{array}{c}-0.120^{*} \\
(0.0678)\end{array}$ & $\begin{array}{c}0.0737 \\
(0.0874)\end{array}$ & $\begin{array}{c}0.0568 \\
(0.0655)\end{array}$ \\
\hline rtightlose_sal & & $\begin{array}{l}0.157^{* *} \\
(0.0708)\end{array}$ & $\begin{array}{l}0.157^{* * *} \\
(0.0503)\end{array}$ & $\begin{array}{l}-0.0174 \\
(0.0410)\end{array}$ & $\begin{array}{l}0.00590 \\
(0.0456)\end{array}$ \\
\hline oldfirm_draw & $\begin{array}{c}0.396^{* * *} \\
(0.101)\end{array}$ & $\begin{array}{c}0.396^{* * *} \\
(0.101)\end{array}$ & $\begin{array}{c}0.396^{* * *} \\
(0.101)\end{array}$ & $\begin{array}{c}0.395^{* * *} \\
(0.101)\end{array}$ & $\begin{array}{c}0.395^{* * *} \\
(0.102)\end{array}$ \\
\hline oldfirm_close & $\begin{array}{l}0.431^{* * *} \\
(0.0509)\end{array}$ & $\begin{array}{l}0.431^{* * *} \\
(0.0510)\end{array}$ & $\begin{array}{l}0.431^{* * *} \\
(0.0510)\end{array}$ & $\begin{array}{l}0.430^{* * *} \\
(0.0510)\end{array}$ & $\begin{array}{l}0.431^{* * *} \\
(0.0511)\end{array}$ \\
\hline oldfirm_rlose & $\begin{array}{l}0.301^{* * *} \\
(0.0443)\end{array}$ & $\begin{array}{l}0.301^{* * *} \\
(0.0444)\end{array}$ & $\begin{array}{l}0.301^{* * *} \\
(0.0444)\end{array}$ & $\begin{array}{l}0.300^{* * *} \\
(0.0443)\end{array}$ & $\begin{array}{l}0.300^{* * *} \\
(0.0445)\end{array}$ \\
\hline cvstr & $\begin{array}{c}-0.0102 \\
(0.0226)\end{array}$ & $\begin{array}{c}-0.0113 \\
(0.0224)\end{array}$ & $\begin{array}{r}-0.00907 \\
(0.0224)\end{array}$ & & $\begin{array}{c}-0.00182 \\
(0.0240)\end{array}$ \\
\hline rustr & $\begin{array}{l}0.00438 \\
(0.0208)\end{array}$ & $\begin{array}{l}0.00281 \\
(0.0196)\end{array}$ & $\begin{array}{c}-0.000433 \\
(0.0213)\end{array}$ & & $\begin{array}{c}-0.00470 \\
(0.0205)\end{array}$ \\
\hline chome & $\begin{array}{c}0.0299 \\
(0.0321)\end{array}$ & $\begin{array}{c}0.0281 \\
(0.0326)\end{array}$ & $\begin{array}{c}0.0418 \\
(0.0330)\end{array}$ & $\begin{array}{c}0.0290 \\
(0.0332)\end{array}$ & $\begin{array}{c}0.0337 \\
(0.0333)\end{array}$ \\
\hline rhome & $\begin{array}{c}0.0167 \\
(0.0320)\end{array}$ & $\begin{array}{c}0.0238 \\
(0.0320)\end{array}$ & $\begin{array}{c}0.0251 \\
(0.0328)\end{array}$ & $\begin{array}{c}0.0188 \\
(0.0320)\end{array}$ & $\begin{array}{c}0.0143 \\
(0.0326)\end{array}$ \\
\hline ctv & $\begin{array}{l}0.0607^{*} \\
(0.0323)\end{array}$ & $\begin{array}{l}0.0568^{*} \\
(0.0323)\end{array}$ & $\begin{array}{l}0.0744^{* *} \\
(0.0321)\end{array}$ & $\begin{array}{l}0.0591^{*} \\
(0.0330)\end{array}$ & $\begin{array}{l}0.0656^{*} \\
(0.0341)\end{array}$ \\
\hline rtv & $\begin{array}{l}0.0455^{*} \\
(0.0268)\end{array}$ & $\begin{array}{l}0.0501^{*} \\
(0.0267)\end{array}$ & $\begin{array}{l}0.0596^{* *} \\
(0.0275)\end{array}$ & $\begin{array}{l}0.0534^{* *} \\
(0.0272)\end{array}$ & $\begin{array}{c}0.0402 \\
(0.0272)\end{array}$ \\
\hline cimp & $\begin{array}{l}0.0758^{*} \\
(0.0443)\end{array}$ & & & $\begin{array}{l}0.0763^{*} \\
(0.0435)\end{array}$ & $\begin{array}{c}0.0716 \\
(0.0461)\end{array}$ \\
\hline rimp & $\begin{array}{c}0.0896 \\
(0.0556)\end{array}$ & & & $\begin{array}{c}0.0775 \\
(0.0549)\end{array}$ & $\begin{array}{c}0.0899 \\
(0.0569)\end{array}$ \\
\hline oldfirm_imp & $\begin{array}{c}0.111 \\
(0.0709) \\
\end{array}$ & $\begin{array}{c}0.111 \\
(0.0711)\end{array}$ & $\begin{array}{c}0.110 \\
(0.0710)\end{array}$ & $\begin{array}{c}0.111 \\
(0.0710) \\
\end{array}$ & $\begin{array}{c}0.112 \\
(0.0711) \\
\end{array}$ \\
\hline intercept & $\begin{array}{l}4.211^{* * *} \\
(0.0139)\end{array}$ & $\begin{array}{l}4.212^{* * *} \\
(0.0139)\end{array}$ & $\begin{array}{l}4.211^{* * *} \\
(0.0139)\end{array}$ & $\begin{array}{l}4.211^{* * *} \\
(0.0139)\end{array}$ & $\begin{array}{l}4.211^{* * * *} \\
(0.0139)\end{array}$ \\
\hline$N$ & 3200 & 3200 & 3200 & 3200 & 3200 \\
\hline
\end{tabular}

Table A.6: OLS regression estimates of specifications B1-B5; dependent variable is the log of the number of domestic violence incidents. 


\section{B Mixed effects model}

As noted in Section 3, one potential drawback of our analysis stems from fans of the two teams under consideration being located in the same region. If, for example, Celtic fans take pleasure from Rangers suffering an unexpected defeat and this leads to a reduction in acts of domestic violence committed by Celtic fans then the effect of the unexpected loss for Rangers will be under-estimated. We argued that it is reasonable to assume that domestic violence would not substantially reduce through such an indirect effect and therefore have assumed that the effect of a disappointing result for a team can be identified. In order to present a further argument to add some justification to this assumption, we can explore a mixed effects model that allows estimates of some carefully chosen parameters to vary across subdivisions. Whilst Glasgow is not a segregated city in terms of the location of football supporters, in some regions support is predominantly in favour of one team, which will allow us to obtain a clearer picture of whether our assumption is reasonable.

The mixed effects Poisson model involves grouping the explanatory variables into those with constant coefficients $\left(Z_{t}\right)$ and those with coefficients which vary across subdivisions $\left(W_{t}\right)$. If $y_{i t}$ is the number of domestic violence incidents in subdivision $i$ on day $t$ then Poisson panel data models assume:

$$
\operatorname{Pr}\left(y_{i t}=y \mid \alpha_{i}\right)=\frac{\exp \left(-\lambda_{i t}\right) \lambda_{i t}^{y}}{y !}
$$

for $y=0,1, \ldots$ The mean is given by:

$$
\lambda_{i t}=\exp \left(Z_{t} \beta+W_{t} \alpha_{i}\right) .
$$

The vector of varying coefficients, $\alpha_{i}$ has a multivariate Normal distribution with diagonal error covariance matrix. Note that mixed effects models can be difficult to estimate precisely when the dimensionality of $\alpha_{i}$ becomes too large. Accordingly, it is common to allow for only a few explanatory variables to have varying coefficients, a practice that we follow here. In particular, we re-estimate model (B1) but allow the coefficients for oldfirm_close, oldfirm_rlose, cfavlose and rfavlose to vary across subdivisions. These are the coefficients that we would expect to vary across subdivisions if there are distinct Rangers (Celtic) neighbourhoods.

In practice, we find that coefficients estimates do not vary much across subdivisions. The average coefficients estimates (i.e. $\beta$ and the mean of $\alpha_{i}$ ) are basically the same as those of model (B1), and so are not presented here. Table A.8 gives estimates (and standard errors) of the deviation of each coefficient 
from the average in each of the 30 police subdivisions using the mixed effects model (estimated using Stata's xtmepoisson command). For two of the variables with subdivision-varying coefficients (oldfirm_close and rfavlose) these deviations are essentially zero. For the remaining two variables (oldfirm_rlose, cfavlose) there is more evidence that their impact varies across subdivision. However, even for these variables, almost all of the estimated deviations are less than one standard deviation from zero and none are more than two standard deviations from zero.

The drawback referred to at the beginning of this section would manifest itself if the correlation between the coefficients on cfavlose and rfavlose or on oldfirm_close and rfavlose, or on oldfirm_rlose and cfavlose were strongly negative. These correlations would mean that in subdivisions where domestic violence increases when Celtic (Rangers) fans receive bad news it tends to reduce when Rangers (Celtic) fans receive bad news. The correlations between the point estimates (ignoring the fact that standard errors tend to be quite large) are given in Table A.7. Given the limited variability across subdivisions of some estimates, we focus on the correlation between oldfirm_rlose and cfavlose: if this is strongly negative then in subdivisions where oldfirm_rlose is higher than average (domestic violence increases more than average when Rangers receive bad news), cfavlose will be less than average, with the implication that Rangers fans might be taking pleasure, and therefore committing fewer acts of domestic violence, when Celtic suffer an upset loss. However, whilst negative, this correlation is quite small and therefore does not suggest that countervailing Rangers and Celtic effects mask each other.

\begin{tabular}{r|cccc} 
& oldfirm_close & oldfirm_rlose & cfavlose & rfavlose \\
\hline oldfirm_close & 1.0000 & & & \\
oldfirm_rlose & 0.3889 & 1.0000 & & \\
cfavlose & -0.3400 & -0.2059 & 1.0000 & \\
rfavlose & -0.2183 & -0.2296 & 0.3952 & 1.0000
\end{tabular}

Table A.7: Correlations across sub-divisions between random effects.

The fact that our results using a mixed effects model on subdivision level data do not indicate substantial variations in coefficients could be due to the scale of police subdivisions. That is, the average police subdivision contains about 75,000 inhabitants and this degree of spatial resolution may be too coarse to pick up effects associated with neighbourhoods of a particular sectarian hue. But, at least the findings of this section are suggestive that our results of Section 5 are not missing important effects due to regional variations within the Strathclyde region. 


\begin{tabular}{|c|c|c|c|c|c|c|c|c|}
\hline \multirow[b]{2}{*}{ subdivision } & \multicolumn{2}{|c|}{ oldfirm_close } & \multicolumn{2}{|c|}{ oldfirm_rlose } & \multicolumn{2}{|c|}{ cfavlose } & \multicolumn{2}{|c|}{ rfavlose } \\
\hline & est & se & est & se & est & se & est & se \\
\hline Average & .4098667 & .0265469 & .2791021 & .0318814 & -.0183021 & .0429036 & -.0241429 & .0379596 \\
\hline 1 & $-3.51 \mathrm{e}-10$ & .0000178 & .0145717 & .0886246 & -.0095377 & .0894032 & $4.19 \mathrm{e}-11$ & $3.16 \mathrm{e}-06$ \\
\hline 2 & $4.80 \mathrm{e}-10$ & .0000178 & -.0409088 & .0907704 & -.0320484 & .0906564 & $3.11 \mathrm{e}-11$ & $3.16 \mathrm{e}-06$ \\
\hline 3 & $-2.25 \mathrm{e}-09$ & .0000178 & .0137195 & .0829717 & -.0188492 & .0857157 & $-8.82 \mathrm{e}-11$ & $3.16 \mathrm{e}-06$ \\
\hline 4 & $-5.77 \mathrm{e}-10$ & .0000178 & -.0761421 & .0756436 & -.0839154 & .0802242 & $1.04 \mathrm{e}-12$ & $3.16 \mathrm{e}-06$ \\
\hline 5 & $1.16 \mathrm{e}-09$ & .0000178 & .0026258 & .075548 & -.0258421 & .0803736 & $-5.05 \mathrm{e}-11$ & $3.16 \mathrm{e}-06$ \\
\hline 6 & $-2.27 \mathrm{e}-09$ & .0000178 & .0157724 & .0774168 & -.0346071 & .0819714 & $7.07 \mathrm{e}-11$ & $3.16 \mathrm{e}-06$ \\
\hline 7 & $2.82 \mathrm{e}-10$ & .0000178 & .0409148 & .0703918 & .0416579 & .0760176 & $-3.17 \mathrm{e}-11$ & $3.16 \mathrm{e}-06$ \\
\hline 8 & $2.57 \mathrm{e}-09$ & .0000178 & -.0749129 & .077453 & .0480438 & .0802122 & $8.06 \mathrm{e}-11$ & $3.16 \mathrm{e}-06$ \\
\hline 9 & $1.11 \mathrm{e}-09$ & .0000178 & .0966942 & .0815901 & -.0189568 & .0854004 & $-1.77 \mathrm{e}-11$ & $3.16 \mathrm{e}-06$ \\
\hline 10 & $-6.59 \mathrm{e}-11$ & .0000178 & .0363059 & .0763423 & -.0023609 & .0810696 & $-5.81 \mathrm{e}-11$ & $3.16 \mathrm{e}-06$ \\
\hline 11 & $-1.93 \mathrm{e}-09$ & .0000178 & .0295256 & .0794474 & -.0098541 & .08332 & $7.16 \mathrm{e}-11$ & $3.16 \mathrm{e}-06$ \\
\hline 12 & $1.71 \mathrm{e}-09$ & .0000178 & .0022421 & .0855686 & -.0097254 & .0873371 & $-2.17 \mathrm{e}-11$ & $3.16 \mathrm{e}-06$ \\
\hline 13 & $-9.01 \mathrm{e}-10$ & .0000178 & -.0970828 & .0815881 & .05686 & .0831927 & $3.59 \mathrm{e}-11$ & $3.16 \mathrm{e}-06$ \\
\hline 14 & $-5.82 \mathrm{e}-13$ & .0000178 & -.0736117 & .0794008 & .0474526 & .0817793 & $-4.90 \mathrm{e}-11$ & $3.16 \mathrm{e}-06$ \\
\hline 15 & $-1.43 \mathrm{e}-09$ & .0000178 & -.0805147 & .0751176 & -.0256493 & .079118 & $-5.56 \mathrm{e}-11$ & $3.16 \mathrm{e}-06$ \\
\hline 16 & $-2.48 \mathrm{e}-09$ & .0000178 & .0571618 & .0777625 & .0284044 & .0820093 & $-2.77 \mathrm{e}-11$ & $3.16 \mathrm{e}-06$ \\
\hline 17 & $1.25 \mathrm{e}-09$ & .0000178 & .023115 & .0776008 & .0039263 & .081798 & $7.74 \mathrm{e}-11$ & $3.16 \mathrm{e}-06$ \\
\hline 18 & $-3.34 \mathrm{e}-09$ & .0000178 & -.0254234 & .0696729 & -.0142166 & .0752988 & $-8.06 \mathrm{e}-11$ & $3.16 \mathrm{e}-06$ \\
\hline 19 & $5.59 \mathrm{e}-10$ & .0000178 & -.0348854 & .0840499 & -.053969 & .086357 & $-7.09 \mathrm{e}-12$ & $3.16 \mathrm{e}-06$ \\
\hline 20 & $5.03 \mathrm{e}-09$ & .0000178 & .1320052 & .0703782 & -.041735 & .0781926 & $-8.76 \mathrm{e}-11$ & $3.16 \mathrm{e}-06$ \\
\hline 21 & $-1.46 \mathrm{e}-09$ & .0000178 & .0287002 & .0801809 & -.0467895 & .0841426 & $3.75 \mathrm{e}-11$ & $3.16 \mathrm{e}-06$ \\
\hline 22 & $-1.73 \mathrm{e}-09$ & .0000178 & -.0463458 & .0822698 & .0236056 & .0844319 & $-6.21 \mathrm{e}-12$ & $3.16 \mathrm{e}-06$ \\
\hline 23 & $2.93 \mathrm{e}-10$ & .0000178 & .0501145 & .0737266 & .0964271 & .0781128 & $6.91 \mathrm{e}-11$ & $3.16 \mathrm{e}-06$ \\
\hline 24 & $3.13 \mathrm{e}-09$ & .0000178 & .0334464 & .0827286 & -.0120527 & .0856436 & $-1.92 \mathrm{e}-11$ & $3.16 \mathrm{e}-06$ \\
\hline 25 & $2.92 \mathrm{e}-09$ & .0000178 & .0095051 & .074862 & -.0304066 & .0799846 & $3.68 \mathrm{e}-12$ & $3.16 \mathrm{e}-06$ \\
\hline 26 & $-1.77 \mathrm{e}-09$ & .0000178 & .0174798 & .0854602 & .0438677 & .0870294 & $3.86 \mathrm{e}-11$ & $3.16 \mathrm{e}-06$ \\
\hline 27 & $1.61 \mathrm{e}-09$ & .0000178 & .0043046 & .081856 & -.009639 & .0848042 & $1.68 \mathrm{e}-11$ & $3.16 \mathrm{e}-06$ \\
\hline 28 & $4.31 \mathrm{e}-09$ & .0000178 & .0711645 & .0675177 & -.0654568 & .0755525 & $-7.93 \mathrm{e}-11$ & $3.16 \mathrm{e}-06$ \\
\hline 29 & $-2.76 \mathrm{e}-09$ & .0000178 & -.0932648 & .0744157 & .0716652 & .0772259 & $4.76 \mathrm{e}-11$ & $3.16 \mathrm{e}-06$ \\
\hline 30 & $-3.12 \mathrm{e}-09$ & .0000178 & -.0073519 & .0730897 & .1064985 & .076761 & $5.66 \mathrm{e}-11$ & $3.16 \mathrm{e}-06$ \\
\hline
\end{tabular}

Table A.8: Mixed effect model estimates of the deviation of coefficients from the average across subdivisions. 